\title{
Tightening the Net on Two Kinds of Dark Matter
}

\section{New dark matter results strengthen constraints on axions and WIMPs.}

\author{
By Marric Stephens
}

$\mathrm{n}$ the continuing game of dark matter hide-and-seek, new results from two experiments have helped the

collaborations behind them rule out further hiding places for the elusive substance $[1,2]$. The results come a year after the team behind another dark matter experiment reported tantalizing signals that could be interpreted as evidence for a hypothesized dark matter particle called an axion (see Viewpoint: Dark Matter Detector Delivers Enigmatic Signal). With neither of the new results indicating the existence of dark matter, the collaborations behind both experiments are now looking to expand their searches and further tighten constraints with future campaigns.

The new results come from the Axion Dark Matter eXperiment (ADMX) at the University of Washington in Seattle [1] and the PandaX-4T experiment at the China Jinping Underground Laboratory (CJPL) [2]. ADMX is a nearly three-decades-old experiment searching for axions and was recently refitted to explore a new, higher range of axion masses. Axions have particular theoretical appeal as a dark matter candidate because they simultaneously explain astronomical

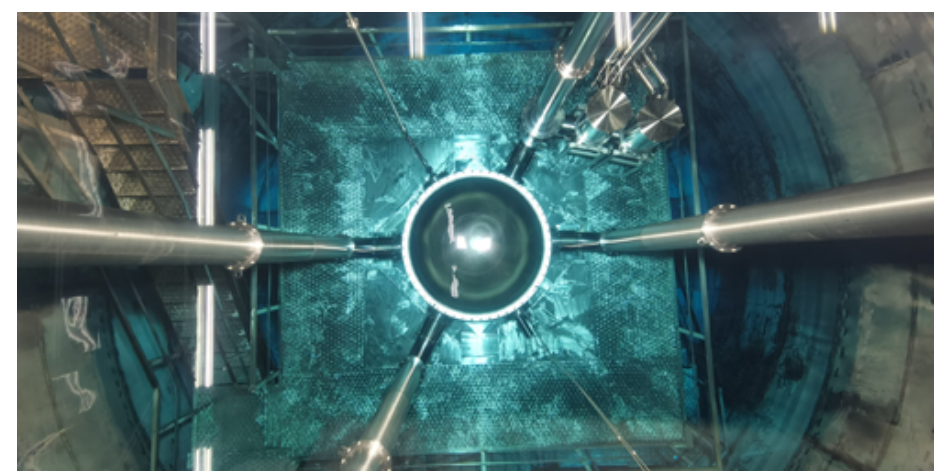

Credit: PandaX observations and solve the strong CP problem-the mystery of why the degree of charge-parity violation in the strong interaction should be so tiny. PandaX-4T is the newest and largest iteration in the Particle and Astrophysical Xenon Detector (PandaX), and it is on the hunt for weakly interacting massive particles (WIMPs).

Analyzing their results, the ADMX Collaboration effectively rules out the existence of axions with energies between 3.3 and 4.2 $\mu \mathrm{eV}$-the range that ADMX is sensitive to-for one axion model (the Kim-Shifman-Vainshtein-Zakharov model). They also strongly constrain the axion-dark-matter density and the axion-photon coupling rate for another axion model (the Dine-Fischler-Srednicki-Zhitnitsky model).

The PandaX-4T Collaboration also finds no dark matter signal in their data, allowing them to claim the most stringent constraint on the interaction rate of WIMPs with nucleons for WIMPs of mass $40 \mathrm{GeV}$. Currently, the collaboration is working to lower the level of background events caused by radioactivity. These changes should allow the team to tighten constraints on WIMPs and also search for the tantalizing axion-like signals found last year.

Marric Stephens is a Corresponding Editor for Physics based in Bristol, UK.

\section{REFERENCES}

1. C. Bartram et al., "Search for invisible axion dark matter in the 3.3-4.2 $\mu \mathrm{eV}$ mass range," Phys. Rev. Lett. 127, 261803 (2021).

2. Y. Meng et al., "Dark matter search results from the PandaX-4T commissioning run,” Phys. Rev. Lett. 127, 261802 (2021). 